\title{
Determinants of Delegation: Evidence from British Establishment Data
}

\author{
Emre Ekinci* \\ Koç University
}

\author{
Nikolaos Theodoropoulos** \\ University of Cyprus
}

\begin{abstract}
This paper provides an empirical analysis of firms' delegation decisions by using a matched employer-employee dataset from Britain. In our analysis, we first investigate if firm-provided training is positively related to delegating authority, as predicted by the existing theories. We then consider variables that can potentially increase the value of delegation. In particular, we conjecture that firms become more likely to delegate authority as they face higher uncertainty or as the employees acquire more precise information. To test these hypotheses, we use market conditions to measure uncertainty and correlates of worker productivity to measure the precision of the employee's information.
\end{abstract}

\section{Yetkilendirme Kararlarının Belirleyicileri: Britanya'daki Firmalar Üzerine Ampirik Analiz}

\begin{abstract}
Özet
Bu çalışma, Britanya'da oluşturulan eşlenmiş işveren-çalışan veri setini kullanarak, firmaların yetkilendirme (delegasyon) kararlarının ampirik bir analizini sunmaktadır. Analizimizde ilk olarak, mevcut teorilerin öngördüğü üzere firma tarafından sağlanan eğitim ile yetkilendirme kararları arasında pozitif bir ilişkili olup olmadığını araştırıyoruz. Daha sonra, yetkilendirmenin değerini potansiyel olarak artırabilecek değişkenleri ele alıyoruz. Özellikle, daha yüksek belirsizlikle karşılaştıklarında veya çalışanların daha kesin bilgiler edinmelerinin mümkün olduğu ortamlarda firmaların yetkilendirme olasılıklarının arttığı hipotezini test ediyoruz. $\mathrm{Bu}$ analizde, belirsizliği ölçmek için piyasa koşullarını kullanırken, çalışanın edinebileceği bilgilerinin kesinliğini ölçmek için ise işçi üretkenliğini etkileyen değişkenleri kullanmaktayız.

Anahtar Kelimeler: Delegasyon; Firma tarafindan verilen eğitim; Britanya firma verileri JEL Sinıflandırması: M12; M53; J01
\end{abstract}

\footnotetext{
* Assistant Professor of Economics, Koç University. Rumelifeneri Yolu, 1, Sarıer, Istanbul- 34450. emekinci@ku.edu.tr. +90 212338 1375. ORCID: 0000-0003-3160-394X

** Associate Professor of Economics, University of Cyprus. P.O. Box 20537, CY-1678 Nicosia, Cyprus. n.theodoropoulos@ucy.ac.cy.00357-22893715. ORCID: 0000-0002-0415-6534
} 
$\mathrm{T}$ he allocation of decision rights to employees is a fundamental challenge that modern firms face. On the one hand, delegating authority to employees leads to the efficient use of scarce resources, such as time or attention, and it reduces the compensation cost as well as motivates the employees (Gibbons et al., 2013). On the other hand, the firm suffers from what Aghion and Tirole (1997) call "loss of control" because while exercising their decision rights, the employees pursue their own interests at the expense of those of the firm. The importance and complexity of delegation decisions have spawned a large body of empirical and theoretical research on the allocation of decision rights. ${ }^{1}$

One strand of the empirical literature focuses on the relationship between incentive pay and delegation. As shown by Prendergast (2002), when the employee has better information than the firm about which actions need to be taken to maximize profits, the firm has stronger incentives to delegate authority to the employee. However, the firm accompanies this delegation of authority with strong incentive pay, so that the employee's opportunistic behavior can be mitigated. Hence, Prendergast's model implies a positive relationship between delegation and incentive pay. Foss and Laursen (2005) and DeVaro and Kurtuluş (2010) provide supporting evidence for this prediction by using data from Danish and British firms, respectively. Departing from the existing literature, DeVaro and Prasad (2015) argue that the relationship between incentives and delegation should vary across occupations; in particular, delegation and incentive pay should be negatively related in complex occupations for which output is a noisy measure of effort. Using data from British firms, they provide evidence in favor of their argument.

Another strand of the literature studies the factors that facilitate the delegation of authority. For example, Bloom et al. (2012) find evidence that firms with more skilled workforce (measured by the proportion of college graduates among all employees) are more likely to delegate authority to their employees. In related work, Acemoglu et al. (2007) find that delegation is more prevalent in heterogenous industries and in firms that are either younger or closer to the technological frontier. Finally, Bilanakos et al. (2018) extend the Aghion-Tirole framework to examine the relationship between delegation and training.

This paper contributes to the empirical literature by examining several determinants of delegation. First, we consider the relationship between the firm's delegation and training decisions. As discussed in the next section, we hypothesize that the level of firm-provided training should be positively related to delegation of authority. Intuitively, when the firm delegates authority to the employee, it has an incentive to facilitate information acquisition prior to the decision making. Thus, under the assumption that firm-provided training induces the employee to exert higher effort for information acquisition, these incentives yield a positive relationship between delegation and training (Bilanakos et al. (2018) and Ekinci and Theodoropoulos (2021)). Next, we turn to the variables that concern the value of information in decision-making. Intuitively, the firm's incentives for delegating authority are strengthened as the value of informed decision-making increases. To this end, we examine first the effect of initial uncertainty in the environment on delegation decisions. Consistent with the theoretical literature (e.g., Prendergast (2002) and Ekinci and Theodoropoulos (2021)), we hypothesize that higher uncertainty is associated with higher propensity to delegating authority. Finally, we consider the precision of information that may be acquired by the employee. Because higher precision raises the value of informed decision-making, there is a positive relationship between delegation and the precision of the employee's signal.

\footnotetext{
${ }^{1}$ See Aghion et al. (2013) and Bolton and Dewatripont (2013) for recent surveys of the literature on the allocation of decision rights.
} 
Overall, we test the following three hypotheses: i) delegation and training are positively related; ii) firms are more likely to delegate authority as the uncertainty in the environment increases; iii) firms are more likely to delegate authority as the worker's signal becomes more precise. To test these hypotheses, we use survey data drawn from the 2004 wave of the Workplace Employee Relations Survey (WERS), a nationally-representative cross-section of British establishments. $^{2}$

Our empirical analysis provides evidence broadly consistent with all three hypotheses. ${ }^{3}$ First, consistent with the first hypothesis, we find strong evidence that higher levels of training are associated with higher likelihood of delegation. Second, we find evidence that firms operating in turbulent markets are more likely to delegate than firms operating in mature markets. This finding supports the second hypothesis, provided that turbulent markets exhibit higher uncertainty relative to mature markets. In addition, we find that younger firms are more likely to delegate. This is also in line with the second hypothesis to the extent that younger firms face greater uncertainty than older firms. Finally, we measure the precision of the employee's information using the employee's age, tenure at the firm, highest educational attainment and the quality of the match between the employee's skills and his or her job. In general, the empirical results concerning these variables are consistent with the third hypothesis. For example, we find that having a college or a postgraduate degree, as opposed to a lower-level degree, is associated with higher likelihood of delegation. Similarly, the empirical results imply that the probability of delegation increases with age and tenure at the firm up to a certain level of age and tenure. Finally, the results reveal that better match between the employee's skills and the job's requirements is associated with higher likelihood of delegation.

The remainder of the paper is organized as follows. We first formulate our testable hypotheses. Then, we describe the data and the key variables used in the empirical analysis. In the last section we present and discuss the empirical results. We conclude the paper with some remarks.

\section{Theoretical Discussion}

Our empirical analysis is largely motivated by Ekinci and Theodoropoulos (2021). To study delegation decisions, Ekinci and Theodoropoulos enrich the standard principal-agent model by incorporating differences of opinion and informal delegation. Following Che and Kartik (2009), they assume that the principal and the agent have differing prior beliefs concerning the state of the world. This implies that although the players observe the same information-if the agent generates a signal - their ex post preferred actions differ since each player interprets the new piece of information differently. In addition to differences of opinion, Ekinci and Theodoropoulos introduce informal delegation, as in Baker et al. (1999), by allowing the principal to retract the agent's authority before exercising it. Their analysis yields the conditions under which the principal finds it optimal to delegate authority to the agent, as opposed to retaining it.

In a part of their analysis, Ekinci and Theodoropoulos (2021) allow the principal to make a costly investment that reduces the agent's cost of information acquisition. The authors argue that the principal's investment can be interpreted as a form of firm-provided training and then show that delegation and training are positively related. Intuitively, the principal has an incentive to provide higher levels of training when the agent is bound to make the decision because the degree of disagreement between the players is mitigated when the decision is made,

\footnotetext{
${ }^{2}$ Other studies that examine the delegation of authority and employ the same data source include Acemoglu et al. (2007), DeVaro and Kurtuluş (2010), DeVaro and Prasad (2015), and Bilanakos et al. (2018).

${ }^{3}$ Note that our empirical results should be interpreted as correlative rather than causal.
} 
by the player with the authority, conditional on observing a signal. Thus, to facilitate information acquisition, the principal provides the agent with higher training. This discussion yields our first hypothesis, which is that delegation and training are positively related. ${ }^{4}$

Two key parameters that alter the principal's delegation decision are the initial uncertainty in the environment and the precision of the signal that may be acquired by the agent. For the intuition, note that higher uncertainty raises the principal's marginal benefit from informed decision-making. The reason is that as the uncertainty increases, the degree of ex post disagreement with the agent becomes less pronounced, and therefore, granting authority to the agent becomes less costly for the principal. ${ }^{5}$ Hence, our second hypothesis is that firms become more likely to delegate authority as the uncertainty in the environment increases. ${ }^{6}$

Finally, we consider how the precision of the signal that may be generated by the agent alters the principal's delegation decision. Intuitively, signal precision and uncertainty in the environment affect the principal's incentives for delegation in the same direction. Specifically, as the agent's potential signal becomes more precise, granting authority to the agent becomes less costly for the principal because the agent's decision, biased from the principal's viewpoint, inflicts less damage on the principal. Hence, our third hypothesis is that firms become more likely to delegate authority as the worker's signal becomes more precise.

Overall, we test the following three hypotheses: i) delegation and training are positively related; ii) firms are more likely to delegate authority as the uncertainty in the environment increases; iii) firms are more likely to delegate authority as the worker's signal becomes more precise.

\section{Description of Data and Key Variables}

Our data is drawn from the 2004 wave of the Workplace Employee Relations Survey (WERS), a large, nationally-representative cross-section of British establishments. ${ }^{7}$ In each wave of the survey, a sample of establishments is randomly drawn from the Interdepartmental Business Register, maintained by the Office of National Statistics. The surveys are stratified by establishment size and industry, with larger workplaces and some industries being overrepresented (Chaplin et al., 2005). The survey population is all British establishments (with 5 or more employees), except those in primary industries and private households with domestic staff. This dataset is suitable for our empirical analysis because it includes information about delegation and training while providing information on a wide range of employee and employer characteristics that can not only serve as important controls but also be used to measure the uncertainty in the environment and the signal precision.

The survey comprises three main sections: 'Management Questionnaire', 'Worker Representative Questionnaire' and 'Employee Questionnaire'. We use information from the management and employee questionnaires. The management questionnaire is administered by a face-to-face interview with the most senior manager who has day-to-day responsibility for industrial relations or personnel matters. For the employee questionnaire, a sample of 25 employees (or all employees if the total number of the employees in the establishment is fewer

\footnotetext{
${ }^{4}$ As shown by Bilanakos et al. (2018), the model analyzed by Aghion and Tirole (1997) yields the same prediction if it is extended to incorporate training as complementary to employee effort.

${ }^{5}$ The authors also show that higher initial uncertainty may lead to higher effort provision under centralization. Even in this case, however, the principal's incentive to delegate authority increases with the uncertainty in the environment as long as the cost of retracting the agent's authority is not large.

${ }^{6}$ Prendergast (2002) derives the same prediction. However, his rationale for the positive relationship between delegation and uncertainty is different. Specifically, he focuses on the monitoring of the agent's actions and shows that under high uncertainty it is costlier for the firm to assign a specific task to the employee than delegating choice of action.

${ }^{7}$ In our discussion we use "workplace", "establishment" and "firm" interchangeably.
} 
than 25) is randomly selected at all establishments participating in the management survey and the questionnaire is self-administered by the employee. ${ }^{8}$ The response rate is $64 \%$ in the management questionnaire and $60 \%$ in the employee questionnaire. Throughout the analysis we use employee weights which correct for the non-response bias and render our working sample representative of the sampling population.

Our delegation measure comes from the employee questionnaire. At each workplace, the surveyed employees are asked: "In general, how much influence do you have about the range of tasks you do in your job?" Responses are recorded on a four-point scale: "None", "A little", "Some" and "A lot". ${ }^{9}$ Despite being subjective, this question is employed to measure the degree of authority being delegated to workers (e.g., DeVaro and Kurtuluş, 2010; DeVaro and Prasad, 2015; Bilanakos et al., 2018). ${ }^{10}$ Our main delegation measure takes on a value of one if the worker's response is "A lot", "Some" or "A little", and zero if the worker's response is "None". ${ }^{11}$ In addition to using a binary variable for delegation, we employ the four distinct answers as a dependent variable in an additional analysis to examine how the degree of delegation is related to the variables of interest.

To measure the amount of training provided to employees at their workplaces, we use the following question from the employee questionnaire: "During the last 12 months how much training have you had, either paid for or organized by your employer: include only training away from your normal place of work, but it could be on or off the premises". The potential responses are "None", "Less than one day", "1 to less than 2 days", "2 to less than 5 days", "5 to less than 10 days" and "10 days or more".

To test the model's implications, we need to measure the uncertainty in the environment and the precision of the worker's signal. For the uncertainty in the environment, we first use binary indicators capturing the current state of the market in which the employee's firm is operating. Specifically, the manager's response indicates whether the market is growing, declining, turbulent or mature. We expect the firm's environment to be more uncertain if the market is turbulent than if it is mature. Further, we use firm age as an alternative proxy for the uncertainty in the environment. In particular, we expect that younger firms face greater uncertainty in their environments (Acemoglu et al. 2007).

For the precision of the employee's signal, we use variables correlated with the worker's productivity. Intuitively, workers can be regarded as more productive either as they become more likely to acquire a signal (holding the cost of acquiring the signal constant) or as their signals become more informative. We conjecture that age, tenure at the firm, and education level are positively correlated with the precision of the signal the worker may acquire. In addition, we measure the match quality between the worker's skills and his or her current job using the following question from the employee questionnaire: "How well do the work skills you personally have match the skills you need to do your present job?". The worker is categorized as overqualified if his response is either "Much higher" or "A bit higher", as qualified if his response is "About the same", and as underqualified if his response is either "A

\footnotetext{
${ }^{8}$ Due to the nature of the multistage sampling procedure used to survey employees, employee questionnaires were distributed only in those establishments where a management interview had taken place.

9 The distribution of responses to this question (after applying employee weights) is as follows: "None" $12.7 \%$, "A little" $14.9 \%$, "Some" $36.9 \%$, and "A lot" $35.4 \%$.

${ }^{10}$ Although Acemoglu et al. (2007) use data from the same source, their unit of analysis is the workplace, and they measure delegation using a question concerning the manager's autonomy from headquarters in making employment decisions.

${ }^{11}$ For robustness we also used an alternative delegation measure which takes a value of one if the worker's response is "A lot" or "Some", and zero otherwise. The results, which are available upon request from the authors, are qualitatively the same as those reported in the paper (see Table 2).
} 
bit lower" or "Much lower". We conjecture that the worker's signal is more precise if he is either overqualified or qualified.

Our empirical analysis excludes establishments which are not in the trading sector (i.e., governmental and non-profit establishments) and those observations for which any variables used in the analysis are missing. Merging the employee questionnaire with the management questionnaire and treating the data at the worker level leaves us with a sample consisting of 10,983 workers clustered in 920 workplaces. Descriptive statistics are reported in Table 1.

Table 1 Descriptive Statistics

\begin{tabular}{|c|c|c|}
\hline Variable & Mean & St. dev. \\
\hline \multicolumn{3}{|l|}{ Individual level variables } \\
\hline $\begin{array}{l}\text { Training } \\
1=" \text { None" } \\
2=\text { ="Less than } 1 \text { day" } \\
3=" 1 \text { to less than } 2 \text { days" } \\
4=" 2 \text { to less than } 5 \text { days" } \\
5=" 5 \text { to less than } 10 \text { days" } \\
6=" 10 \text { days or more" }\end{array}$ & $\begin{array}{l}0.394 \\
0.109 \\
0.140 \\
0.200 \\
0.085 \\
0.073\end{array}$ & $\begin{array}{l}0.489 \\
0.311 \\
0.347 \\
0.400 \\
0.278 \\
0.261\end{array}$ \\
\hline $\begin{array}{l}\text { Personal characteristics } \\
\text { Female } \\
\text { Male } \\
\text { Age }\end{array}$ & $\begin{array}{c}0.472 \\
0.528 \\
39.054\end{array}$ & $\begin{array}{c}0.499 \\
0.499 \\
12.444\end{array}$ \\
\hline $\begin{array}{l}\text { Job characteristics } \\
\text { Tenure } \\
\text { Permanent job } \\
\text { Temporary job } \\
\text { Fixed term job }\end{array}$ & $\begin{array}{l}7.144 \\
0.923 \\
0.050 \\
0.027\end{array}$ & $\begin{array}{l}7.317 \\
0.267 \\
0.218 \\
0.163\end{array}$ \\
\hline $\begin{array}{l}\text { Occupations } \\
\text { Managers, senior administrators } \\
\text { Professional } \\
\text { Associate professional, technical } \\
\text { Clerical and secretarial } \\
\text { Craft and skilled service } \\
\text { Personal and protective service } \\
\text { Sales } \\
\text { Operative and assembly } \\
\text { Routine/unskilled }\end{array}$ & $\begin{array}{l}0.102 \\
0.092 \\
0.132 \\
0.162 \\
0.084 \\
0.059 \\
0.111 \\
0.108 \\
0.149\end{array}$ & $\begin{array}{l}0.303 \\
0.289 \\
0.339 \\
0.369 \\
0.277 \\
0.235 \\
0.315 \\
0.310 \\
0.356\end{array}$ \\
\hline $\begin{array}{l}\text { Academic qualifications } \\
\text { GCSE, grades D-G } \\
\text { GCSE, grades A-C } \\
\text { A levels (1 A-level and above) } \\
\text { First degree (BSc, BA, Bed, HND, HNC) } \\
\text { Higher degree or equivalent (MSc, MA, MBA, PGCE, PhD) } \\
\text { No educational qualification or other educational qualification }\end{array}$ & $\begin{array}{l}0.081 \\
0.173 \\
0.093 \\
0.125 \\
0.040 \\
0.488\end{array}$ & $\begin{array}{l}0.272 \\
0.378 \\
0.291 \\
0.331 \\
0.195 \\
0.500\end{array}$ \\
\hline $\begin{array}{l}\text { Job-skill match } \\
\text { Overqualified } \\
\text { Qualified } \\
\text { Underqualified }\end{array}$ & $\begin{array}{l}0.543 \\
0.409 \\
0.047\end{array}$ & $\begin{array}{l}0.498 \\
0.492 \\
0.212\end{array}$ \\
\hline \multicolumn{3}{|l|}{ Workplace level variables } \\
\hline $\begin{array}{l}\text { Manufacturing } \\
\text { Utilities (electricity, gas, water) } \\
\text { Construction } \\
\text { Wholesale and retail } \\
\text { Hotels and restaurants }\end{array}$ & $\begin{array}{l}0.156 \\
0.001 \\
0.042 \\
0.229 \\
0.065\end{array}$ & $\begin{array}{l}0.363 \\
0.037 \\
0.200 \\
0.420 \\
0.246\end{array}$ \\
\hline
\end{tabular}




\begin{tabular}{|c|c|c|}
\hline Transportation and communication & 0.056 & 0.230 \\
\hline Financial services & 0.063 & 0.243 \\
\hline Other business services & 0.171 & 0.376 \\
\hline Education & 0.013 & 0.115 \\
\hline Health & 0.141 & 0.348 \\
\hline Other community services & 0.063 & 0.243 \\
\hline \multicolumn{3}{|l|}{ Region } \\
\hline Scotland & 0.088 & 0.284 \\
\hline North & 0.058 & 0.235 \\
\hline North west & 0.139 & 0.346 \\
\hline East Midlands & 0.075 & 0.263 \\
\hline West Midlands & 0.11 & 0.313 \\
\hline East Anglia & 0.054 & 0.226 \\
\hline South east & 0.258 & 0.437 \\
\hline South west & 0.094 & 0.291 \\
\hline Wales & 0.028 & 0.166 \\
\hline Yorkshire and Humberside & 0.095 & 0.293 \\
\hline \multicolumn{3}{|l|}{ Workplace characteristics } \\
\hline Number of employees in the workplace & 55.930 & 136.197 \\
\hline Part of a larger organization & 0.617 & 0.486 \\
\hline Single independent workplace not belonging & 0.366 & 0.482 \\
\hline Sole UK establishment of a foreign organization & 0.017 & 0.130 \\
\hline Workplace age - less than 5 years & 0.091 & 0.287 \\
\hline Workplace age - 5 to 9 years & 0.126 & 0.332 \\
\hline Workplace age - 10 to 14 years & 0.137 & 0.344 \\
\hline Workplace age -15 to 20 years & 0.173 & 0.378 \\
\hline Workplace age - 21 to 24 years & 0.061 & 0.239 \\
\hline Workplace age - 25 years plus & 0.413 & 0.492 \\
\hline \multicolumn{3}{|l|}{ Occupational group percentages } \\
\hline Managerial and senior administrative & 0.125 & 0.097 \\
\hline Professional & 0.071 & 0.164 \\
\hline Technical & 0.071 & 0.151 \\
\hline Sales & 0.181 & 0.304 \\
\hline Operative and assembly & 0.109 & 0.221 \\
\hline Clerical and secretarial & 0.146 & 0.207 \\
\hline Craft and skilled services & 0.086 & 0.188 \\
\hline Protective and personal service & 0.092 & 0.244 \\
\hline Routine/unskilled & 0.117 & 0.232 \\
\hline \multicolumn{3}{|l|}{ Current state of the market } \\
\hline Market is growing & 0.508 & 0.500 \\
\hline Market is declining & 0.105 & 0.306 \\
\hline Market is turbulent & 0.143 & 0.350 \\
\hline Market is mature & 0.244 & 0.429 \\
\hline \multicolumn{3}{|l|}{ Number of competitors } \\
\hline No competitors & 0.066 & 0.249 \\
\hline Few competitors (less than 5 ) & 0.386 & 0.487 \\
\hline Many competitors & 0.547 & 0.498 \\
\hline
\end{tabular}

Notes: This table displays the descriptive statistics for all variables used in the analysis. Individual characteristics are weighted using employment weights and establishment characteristics are weighted using establishment weights.

\section{Discussion of Empirical Results}

To test our hypotheses, we first estimate probit models where the dependent variable is an indicator variable that takes a value of one if the worker is granted authority and zero if not. ${ }^{12}$

\footnotetext{
12 Throughout the empirical analysis, standard errors are clustered at the workplace level.
} 
The results from probit models are reported in Table 2 and the corresponding marginal effects are reported in Table $3 .^{13}$

\section{Table 2 Determinants of Delegation}

(Dependent Variable: Delegation (0=“no delegation”, 1=“delegation”))

\begin{tabular}{|c|c|c|c|c|c|c|}
\hline VARIABLES & $(1)$ & $(2)$ & (3) & $(4)$ & $(5)$ & $(6)$ \\
\hline Training & $\begin{array}{c}0.228 * * * \\
(0.034)\end{array}$ & $\begin{array}{c}0.157 * * * \\
(0.036)\end{array}$ & $\begin{array}{c}0.155^{* * * *} \\
(0.036)\end{array}$ & & & \\
\hline \multicolumn{7}{|l|}{ Training } \\
\hline$<1$ day & & & & $\begin{array}{c}0.053 \\
(0.055)\end{array}$ & $\begin{array}{c}0.060 \\
(0.055)\end{array}$ & $\begin{array}{c}0.063 \\
(0.055)\end{array}$ \\
\hline$>=1$ day to $<2$ days & & & & $\begin{array}{c}0.230 * * * \\
(0.053)\end{array}$ & $\begin{array}{c}0.176 * * * \\
(0.054)\end{array}$ & $\begin{array}{c}0.171 * * * \\
(0.054)\end{array}$ \\
\hline$>=2$ days to $<5$ days & & & & $\begin{array}{c}0.332 * * * \\
(0.048)\end{array}$ & $\begin{array}{c}0.235^{* * * *} \\
(0.050)\end{array}$ & $\begin{array}{c}0.233 * * * \\
(0.050)\end{array}$ \\
\hline$>=5$ days to $<10$ days & & & & $\begin{array}{c}0.292 * * * \\
(0.067)\end{array}$ & $\begin{array}{c}0.149 * * \\
(0.069)\end{array}$ & $\begin{array}{c}0.150 * * \\
(0.069)\end{array}$ \\
\hline$>=10$ days & & & & $\begin{array}{c}0.219 * * * \\
(0.065)\end{array}$ & $\begin{array}{c}0.130 * * \\
(0.066)\end{array}$ & $\begin{array}{l}0.125^{*} \\
(0.066)\end{array}$ \\
\hline Growing market & $\begin{array}{c}0.072 \\
(0.047)\end{array}$ & $\begin{array}{c}0.080 * \\
(0.046)\end{array}$ & $\begin{array}{c}0.061 \\
(0.046)\end{array}$ & $\begin{array}{c}0.071 \\
(0.046)\end{array}$ & $\begin{array}{l}0.079 * \\
(0.046)\end{array}$ & $\begin{array}{c}0.061 \\
(0.046)\end{array}$ \\
\hline Declining market & $\begin{array}{c}0.057 \\
(0.079)\end{array}$ & $\begin{array}{c}0.068 \\
(0.078)\end{array}$ & $\begin{array}{c}0.038 \\
(0.080)\end{array}$ & $\begin{array}{c}0.055 \\
(0.079)\end{array}$ & $\begin{array}{c}0.066 \\
(0.078)\end{array}$ & $\begin{array}{c}0.036 \\
(0.080)\end{array}$ \\
\hline Turbulent market & $\begin{array}{c}0.118 * * \\
(0.054)\end{array}$ & $\begin{array}{c}0.126 * * \\
(0.054)\end{array}$ & $\begin{array}{l}0.099 * \\
(0.053)\end{array}$ & $\begin{array}{c}0.119 * * \\
(0.054)\end{array}$ & $\begin{array}{c}0.126^{* *} \\
(0.054)\end{array}$ & $\begin{array}{l}0.100 * \\
(0.053)\end{array}$ \\
\hline GCSE grades D-G & $\begin{array}{l}-0.041 \\
(0.061)\end{array}$ & $\begin{array}{c}0.035 \\
(0.063)\end{array}$ & $\begin{array}{c}0.031 \\
(0.063)\end{array}$ & $\begin{array}{l}-0.041 \\
(0.061)\end{array}$ & $\begin{array}{c}0.033 \\
(0.063)\end{array}$ & $\begin{array}{c}0.030 \\
(0.063)\end{array}$ \\
\hline GCSE grades A-C & $\begin{array}{l}-0.021 \\
(0.042)\end{array}$ & $\begin{array}{l}-0.021 \\
(0.045)\end{array}$ & $\begin{array}{l}-0.022 \\
(0.045)\end{array}$ & $\begin{array}{l}-0.019 \\
(0.042)\end{array}$ & $\begin{array}{l}-0.022 \\
(0.045)\end{array}$ & $\begin{array}{l}-0.022 \\
(0.045)\end{array}$ \\
\hline A-levels & $\begin{array}{c}0.085 \\
(0.058)\end{array}$ & $\begin{array}{c}0.026 \\
(0.060)\end{array}$ & $\begin{array}{c}0.019 \\
(0.061)\end{array}$ & $\begin{array}{c}0.082 \\
(0.058)\end{array}$ & $\begin{array}{c}0.024 \\
(0.060)\end{array}$ & $\begin{array}{c}0.018 \\
(0.061)\end{array}$ \\
\hline First degree & $\begin{array}{c}0.223 * * * \\
(0.059)\end{array}$ & $\begin{array}{c}0.049 \\
(0.062)\end{array}$ & $\begin{array}{c}0.038 \\
(0.062)\end{array}$ & $\begin{array}{c}0.216 * * * \\
(0.060)\end{array}$ & $\begin{array}{c}0.047 \\
(0.063)\end{array}$ & $\begin{array}{c}0.036 \\
(0.062)\end{array}$ \\
\hline Higher degree & $\begin{array}{c}0.315 * * * \\
(0.101)\end{array}$ & $\begin{array}{c}0.094 \\
(0.106)\end{array}$ & $\begin{array}{c}0.085 \\
(0.107)\end{array}$ & $\begin{array}{c}0.309 * * * \\
(0.102)\end{array}$ & $\begin{array}{c}0.092 \\
(0.107)\end{array}$ & $\begin{array}{c}0.084 \\
(0.107)\end{array}$ \\
\hline Age & $\begin{array}{c}0.040 * * * \\
(0.009)\end{array}$ & $\begin{array}{c}0.027 * * * \\
(0.009)\end{array}$ & $\begin{array}{c}0.027 * * * \\
(0.009)\end{array}$ & $\begin{array}{c}0.039 * * * \\
(0.009)\end{array}$ & $\begin{array}{c}0.026 * * * \\
(0.009)\end{array}$ & $\begin{array}{c}0.026^{* * *} \\
(0.009)\end{array}$ \\
\hline Age sq/100 & $\begin{array}{c}-0.053 * * * \\
(0.011)\end{array}$ & $\begin{array}{c}-0.037 * * * \\
(0.011)\end{array}$ & $\begin{array}{c}-0.036 * * * * \\
(0.011)\end{array}$ & $\begin{array}{c}-0.051 * * * \\
(0.011)\end{array}$ & $\begin{array}{c}-0.036 * * * \\
(0.011)\end{array}$ & $\begin{array}{c}-0.035 * * * \\
(0.011)\end{array}$ \\
\hline Tenure & $\begin{array}{c}0.042 * * * \\
(0.012)\end{array}$ & $\begin{array}{c}0.036 * * * \\
(0.012)\end{array}$ & $\begin{array}{c}0.037 * * * \\
(0.012)\end{array}$ & $\begin{array}{c}0.042 * * * \\
(0.012)\end{array}$ & $\begin{array}{c}0.036 * * * \\
(0.012)\end{array}$ & $\begin{array}{c}0.037 * * * \\
(0.012)\end{array}$ \\
\hline Tenure sq/100 & $\begin{array}{c}-0.101 * \\
(0.054)\end{array}$ & $\begin{array}{l}-0.079 \\
(0.055)\end{array}$ & $\begin{array}{l}-0.085 \\
(0.055)\end{array}$ & $\begin{array}{c}-0.101 * \\
(0.054)\end{array}$ & $\begin{array}{l}-0.078 \\
(0.055)\end{array}$ & $\begin{array}{l}-0.084 \\
(0.055)\end{array}$ \\
\hline Overqualified & $\begin{array}{c}0.158 * * \\
(0.072)\end{array}$ & $\begin{array}{c}0.221 * * * \\
(0.073)\end{array}$ & $\begin{array}{c}0.225 * * * \\
(0.073)\end{array}$ & $\begin{array}{c}0.158 * * \\
(0.072)\end{array}$ & $\begin{array}{c}0.218 * * * \\
(0.073)\end{array}$ & $\begin{array}{c}0.221 * * * \\
(0.073)\end{array}$ \\
\hline Qualified & $\begin{array}{c}0.280 * * * \\
(0.074)\end{array}$ & $\begin{array}{c}0.318 * * * \\
(0.076)\end{array}$ & $\begin{array}{c}0.324 * * * \\
(0.076)\end{array}$ & $\begin{array}{c}0.272 * * * \\
(0.074)\end{array}$ & $\begin{array}{c}0.311 * * * \\
(0.076)\end{array}$ & $\begin{array}{c}0.317 * * * \\
(0.076)\end{array}$ \\
\hline Female & $\begin{array}{c}-0.142 * * * \\
(0.038)\end{array}$ & $\begin{array}{c}-0.161 * * * \\
(0.041)\end{array}$ & $\begin{array}{c}-0.154 * * * \\
(0.041)\end{array}$ & $\begin{array}{c}-0.135 * * * \\
(0.039)\end{array}$ & $\begin{array}{c}-0.158 * * * \\
(0.041)\end{array}$ & $\begin{array}{c}-0.151 * * * \\
(0.041)\end{array}$ \\
\hline Permanent job & $\begin{array}{c}-0.036 \\
(0.096)\end{array}$ & $\begin{array}{c}0.023 \\
(0.103)\end{array}$ & $\begin{array}{c}0.027 \\
(0.104)\end{array}$ & $\begin{array}{l}-0.037 \\
(0.095)\end{array}$ & $\begin{array}{c}0.021 \\
(0.102)\end{array}$ & $\begin{array}{c}0.024 \\
(0.103)\end{array}$ \\
\hline Temporary job & $\begin{array}{c}-0.192 \\
(0.118) \\
\end{array}$ & $\begin{array}{l}-0.061 \\
(0.125) \\
\end{array}$ & $\begin{array}{l}-0.050 \\
(0.127)\end{array}$ & $\begin{array}{c}-0.175 \\
(0.118) \\
\end{array}$ & $\begin{array}{l}-0.057 \\
(0.124) \\
\end{array}$ & $\begin{array}{l}-0.048 \\
(0.125)\end{array}$ \\
\hline Log no. of employees & $\begin{array}{c}-0.044 * * * \\
(0.015)\end{array}$ & $\begin{array}{c}-0.043 * * * \\
(0.015)\end{array}$ & $\begin{array}{c}-0.040 * * \\
(0.017)\end{array}$ & $\begin{array}{c}-0.044 * * * \\
(0.015)\end{array}$ & $\begin{array}{c}-0.042 * * * \\
(0.015)\end{array}$ & $\begin{array}{c}-0.040 * * \\
(0.017)\end{array}$ \\
\hline
\end{tabular}

\footnotetext{
${ }^{13}$ To calculate the marginal effect of a variable on the probability of delegation, other explanatory variables are set equal to their means in the sample.
} 


\begin{tabular}{|c|c|c|c|c|c|c|}
\hline Part of a larger workplace & $\begin{array}{c}-0.239 * * \\
(0.102)\end{array}$ & $\begin{array}{c}-0.158 \\
(0.108)\end{array}$ & $\begin{array}{l}-0.157 \\
(0.115)\end{array}$ & $\begin{array}{c}-0.250 * * \\
(0.100)\end{array}$ & $\begin{array}{l}-0.166 \\
(0.106)\end{array}$ & $\begin{array}{l}-0.166 \\
(0.114)\end{array}$ \\
\hline \multirow[t]{2}{*}{ Single independent workplace } & -0.151 & -0.033 & -0.022 & -0.160 & -0.041 & -0.030 \\
\hline & $(0.107)$ & $(0.113)$ & $(0.121)$ & $(0.105)$ & $(0.112)$ & $(0.120)$ \\
\hline \multirow[t]{2}{*}{ Workplace age $<5$ years } & $0.182 * *$ & $0.172 *$ & $0.175 * *$ & $0.182 * *$ & $0.170^{*}$ & $0.174 * *$ \\
\hline & $(0.090)$ & $(0.092)$ & $(0.086)$ & $(0.091)$ & $(0.092)$ & $(0.085)$ \\
\hline \multirow{2}{*}{ Workplace age $>=5$ to $<=9$} & $0.132 * *$ & $0.131 * *$ & $0.135 * *$ & $0.130 * *$ & $0.130 * *$ & $0.134 * *$ \\
\hline & $(0.064)$ & $(0.062)$ & $(0.062)$ & $(0.063)$ & $(0.062)$ & $(0.062)$ \\
\hline \multirow[t]{2}{*}{ Workplace age $>=10$ to $<=14$} & 0.009 & 0.051 & 0.065 & 0.012 & 0.052 & 0.065 \\
\hline & $(0.061)$ & $(0.063)$ & $(0.064)$ & $(0.061)$ & $(0.063)$ & $(0.064)$ \\
\hline \multirow[t]{2}{*}{ Workplace age $>=15$ to $<=20$} & 0.041 & 0.045 & 0.045 & 0.044 & 0.045 & 0.045 \\
\hline & $(0.056)$ & $(0.055)$ & $(0.054)$ & $(0.055)$ & $(0.055)$ & $(0.053)$ \\
\hline \multirow{2}{*}{ Workplace age $>=21$ to $<=24$} & 0.122 & 0.137 & $0.144 *$ & 0.122 & 0.135 & $0.142 *$ \\
\hline & $(0.085)$ & $(0.086)$ & $(0.085)$ & $(0.086)$ & $(0.086)$ & $(0.085)$ \\
\hline \multirow[t]{2}{*}{ Few competitors $(<5)$} & -0.126 & -0.118 & $-0.161 * *$ & -0.122 & -0.115 & $-0.158 * *$ \\
\hline & $(0.085)$ & $(0.088)$ & $(0.078)$ & $(0.085)$ & $(0.088)$ & $(0.078)$ \\
\hline \multirow[t]{2}{*}{ Many competitors } & -0.134 & -0.139 & $-0.177 * *$ & -0.132 & -0.138 & $-0.175 * *$ \\
\hline & $(0.083)$ & $(0.086)$ & $(0.076)$ & $(0.083)$ & $(0.086)$ & $(0.076)$ \\
\hline \multirow[t]{2}{*}{ Manager } & & $1.188 * * *$ & $1.192 * * *$ & & $1.170 * * *$ & $1.175^{* * *}$ \\
\hline & & $(0.110)$ & $(0.114)$ & & $(0.110)$ & $(0.113)$ \\
\hline \multirow[t]{2}{*}{ Professional } & & $0.668 * * *$ & $0.676^{* * *}$ & & $0.657 * * *$ & $0.666 * * *$ \\
\hline & & $(0.084)$ & $(0.090)$ & & $(0.084)$ & $(0.090)$ \\
\hline \multirow[t]{2}{*}{ Technical } & & $0.587 * * *$ & $0.596 * * *$ & & $0.573 * * *$ & $0.583 * * *$ \\
\hline & & $(0.074)$ & $(0.077)$ & & $(0.074)$ & $(0.076)$ \\
\hline \multirow[t]{2}{*}{ Clerk } & & $0.300 * * *$ & $0.332 * * *$ & & $0.291 * * *$ & $0.324 * * *$ \\
\hline & & $(0.068)$ & $(0.069)$ & & $(0.068)$ & $(0.069)$ \\
\hline \multirow[t]{2}{*}{ Craft } & & 0.080 & 0.105 & & 0.074 & 0.101 \\
\hline & & $(0.072)$ & $(0.077)$ & & $(0.073)$ & $(0.077)$ \\
\hline \multirow[t]{2}{*}{ Service } & & 0.120 & 0.142 & & 0.113 & 0.137 \\
\hline & & $(0.085)$ & $(0.091)$ & & $(0.085)$ & $(0.092)$ \\
\hline \multirow[t]{2}{*}{ Sales } & & $0.138 *$ & $0.136^{*}$ & & $0.130^{*}$ & 0.130 \\
\hline & & $(0.075)$ & $(0.081)$ & & $(0.075)$ & $(0.081)$ \\
\hline \multirow[t]{2}{*}{ Operative } & & -0.064 & -0.093 & & -0.069 & -0.097 \\
\hline & & $(0.065)$ & $(0.071)$ & & $(0.065)$ & $(0.071)$ \\
\hline Industry dummies & Yes & Yes & Yes & Yes & Yes & Yes \\
\hline Region dummies & Yes & Yes & Yes & Yes & Yes & Yes \\
\hline Workforce composition & No & No & Yes & No & No & Yes \\
\hline \multirow[t]{2}{*}{ Constant } & $0.462 *$ & 0.307 & 0.376 & $0.490^{*}$ & 0.337 & 0.408 \\
\hline & $(0.268)$ & $(0.277)$ & $(0.290)$ & $(0.265)$ & $(0.273)$ & $(0.286)$ \\
\hline
\end{tabular}

Notes: Cell entries are probit coefficients with standard errors clustered at the workplace level. The omitted categories are: male, no training, no academic or other educational qualification, underqualified, routine/unskilled occupation, fixed term job, percentage of routine/unskilled staff, mature market, no competitors, sole UK workplace of a foreign organization, workplace older than 25 years of age, other community services sector, Yorkshire and Humberside". Levels of significance: $* * * \mathrm{p}<0.01, * * \mathrm{p}<0.05, * \mathrm{p}<0.1$.

Our baseline specification, reported in column 1, includes a binary variable for training, the worker's age and tenure (both with squared terms), indicator variables for education (the omitted category is "no academic qualifications or other qualification"), job-skill match (the omitted category is "underqualified"), and indicators capturing the current state of the market in which the firm is operating (the omitted category is "operating in a mature market"). In addition, the baseline specification includes the following controls: firm size (the log of the number of employees), indicators for how many years the firm has been operating (the omitted category is "more than 25 years"), if the worker has a permanent or a temporary job with no agreed end date (the omitted category is having a fixed-period job with an agreed end date), whether the workplace is a part of a larger organization (the omitted category is "sole UK establishment of a foreign organization"), and finally indicators for industry and region. In column 2, we add indicators for the worker's occupation (manager, professional, associate 
professional and technical, clerical and secretarial, craft and skilled service, personal and protective service, sales, plant and machine operatives, where the omitted category is "other occupation"). In column 3, we add indicator variables capturing the occupational composition of the workforce (i.e., the percentage of: managers, professional staff, technical staff, clerical staff, craft (skilled) staff, service staff and sales staff, where the omitted category is the share of "other occupations"). ${ }^{14}$ The specifications in columns 4 through 6 are the same as those in columns 1 through 3 , respectively, except in the latter set of specifications, we use six categories, rather than a binary variable, for the amount of training received by the worker. In these latter specifications, the omitted category for training is "None".

Table 3 Marginal Effects on Probability of Delegation

\begin{tabular}{|c|c|c|c|c|c|c|}
\hline VARIABLES & (1) & $(2)$ & (3) & (4) & (5) & (6) \\
\hline Training & $\begin{array}{c}0.041 * * * \\
(0.006)\end{array}$ & $\begin{array}{c}0.027 * * * \\
(0.006)\end{array}$ & $\begin{array}{c}0.026 * * * \\
(0.006)\end{array}$ & & & \\
\hline \multicolumn{7}{|l|}{ Training } \\
\hline$<1$ day & & & & $\begin{array}{c}0.007 \\
(0.009)\end{array}$ & $\begin{array}{c}0.008 \\
(0.008)\end{array}$ & $\begin{array}{c}0.009 \\
(0.009)\end{array}$ \\
\hline$>=1$ day to $<2$ days & & & & $\begin{array}{c}0.035 * * * \\
(0.008)\end{array}$ & $\begin{array}{c}0.025 * * * \\
(0.008)\end{array}$ & $\begin{array}{c}0.025 * * * \\
(0.008)\end{array}$ \\
\hline$>=2$ days to $<5$ days & & & & $\begin{array}{c}0.051 * * * \\
(0.007)\end{array}$ & $\begin{array}{c}0.035 * * * \\
(0.007)\end{array}$ & $\begin{array}{c}0.035^{* * *} * \\
(0.007)\end{array}$ \\
\hline$>=5$ days to $<10$ days & & & & $\begin{array}{c}0.049 * * * \\
(0.008)\end{array}$ & $\begin{array}{c}0.030 * * * \\
(0.009)\end{array}$ & $\begin{array}{c}0.030 * * * \\
(0.009)\end{array}$ \\
\hline$>=10$ days & & & & $\begin{array}{c}0.033 * * * \\
(0.009)\end{array}$ & $\begin{array}{c}0.019 * * \\
(0.009) \\
\end{array}$ & $\begin{array}{l}0.019 * \\
(0.009) \\
\end{array}$ \\
\hline Growing market & $\begin{array}{c}0.014 \\
(0.009)\end{array}$ & $\begin{array}{l}0.014 * \\
(0.008)\end{array}$ & $\begin{array}{c}0.011 \\
(0.007)\end{array}$ & $\begin{array}{l}0.014 * \\
(0.008)\end{array}$ & $\begin{array}{l}0.014 * \\
(0.008)\end{array}$ & $\begin{array}{c}0.011 \\
(0.007)\end{array}$ \\
\hline Declining market & $\begin{array}{c}0.008 \\
(0.013)\end{array}$ & $\begin{array}{c}0.008 \\
(0.011)\end{array}$ & $\begin{array}{c}0.003 \\
(0.012)\end{array}$ & $\begin{array}{c}0.007 \\
(0.013)\end{array}$ & $\begin{array}{c}0.007 \\
(0.011)\end{array}$ & $\begin{array}{c}0.003 \\
(0.012)\end{array}$ \\
\hline Turbulent market & $\begin{array}{c}0.019 * * \\
(0.009)\end{array}$ & $\begin{array}{c}0.018 * * \\
(0.008)\end{array}$ & $\begin{array}{l}0.015 * \\
(0.008) \\
\end{array}$ & $\begin{array}{c}0.019 * * \\
(0.008)\end{array}$ & $\begin{array}{c}0.018 * * \\
(0.008) \\
\end{array}$ & $\begin{array}{l}0.016 * \\
(0.008) \\
\end{array}$ \\
\hline GCSE grades D-G & $\begin{array}{c}0.016 \\
(0.011)\end{array}$ & $\begin{array}{c}0.012 \\
(0.011)\end{array}$ & $\begin{array}{c}0.012 \\
(0.011)\end{array}$ & $\begin{array}{c}0.016 \\
(0.011)\end{array}$ & $\begin{array}{c}0.012 \\
(0.010)\end{array}$ & $\begin{array}{c}0.012 \\
(0.010)\end{array}$ \\
\hline GCSE grades A-C & $\begin{array}{c}0.023^{* * *} * \\
(0.008)\end{array}$ & $\begin{array}{c}0.006 \\
(0.009)\end{array}$ & $\begin{array}{c}0.007 \\
(0.009)\end{array}$ & $\begin{array}{c}0.023 * * * \\
(0.008)\end{array}$ & $\begin{array}{c}0.007 \\
(0.009)\end{array}$ & $\begin{array}{c}0.007 \\
(0.009)\end{array}$ \\
\hline A-levels & $\begin{array}{c}0.040 * * * \\
(0.009)\end{array}$ & $\begin{array}{c}0.015 \\
(0.011)\end{array}$ & $\begin{array}{c}0.015 \\
(0.010)\end{array}$ & $\begin{array}{c}0.039 * * * \\
(0.009)\end{array}$ & $\begin{array}{c}0.015 \\
(0.011)\end{array}$ & $\begin{array}{c}0.015 \\
(0.010)\end{array}$ \\
\hline First degree & $\begin{array}{c}0.049 * * * \\
(0.008)\end{array}$ & $\begin{array}{l}0.016^{*} \\
(0.009)\end{array}$ & $\begin{array}{l}0.016^{*} \\
(0.009)\end{array}$ & $\begin{array}{c}0.048 * * * \\
(0.008)\end{array}$ & $\begin{array}{l}0.016^{*} \\
(0.008)\end{array}$ & $\begin{array}{l}0.016^{*} \\
(0.008)\end{array}$ \\
\hline Higher degree & $\begin{array}{c}0.0065 * * * \\
(0.010)\end{array}$ & $\begin{array}{l}0.025^{*} \\
(0.014)\end{array}$ & $\begin{array}{c}0.024 \\
(0.016) \\
\end{array}$ & $\begin{array}{c}0.063 * * * \\
(0.010)\end{array}$ & $\begin{array}{c}0.025 \\
(0.016) \\
\end{array}$ & $\begin{array}{c}0.024 \\
(0.016)\end{array}$ \\
\hline Age & $\begin{array}{c}0.007 * * * \\
(0.001)\end{array}$ & $\begin{array}{c}0.005 * * * \\
(0.001)\end{array}$ & $\begin{array}{c}0.005 * * * \\
(0.001)\end{array}$ & $\begin{array}{c}0.007 * * * \\
(0.001)\end{array}$ & $\begin{array}{c}0.004 * * * \\
(0.002)\end{array}$ & $\begin{array}{c}0.004 * * * \\
(0.002)\end{array}$ \\
\hline Age sq/100 & $\begin{array}{c}-0.009 * * * \\
(0.001)\end{array}$ & $\begin{array}{c}-0.006^{* * * *} \\
(0.002)\end{array}$ & $\begin{array}{c}-0.006 * * * \\
(0.001)\end{array}$ & $\begin{array}{c}-0.009 * * * \\
(0.002)\end{array}$ & $\begin{array}{c}-0.006 * * * \\
(0.002)\end{array}$ & $\begin{array}{c}-0.005 * * * \\
(0.002)\end{array}$ \\
\hline Tenure & $\begin{array}{c}0.008 * * * \\
(0.002)\end{array}$ & $\begin{array}{c}0.006 * * * \\
(0.003)\end{array}$ & $\begin{array}{c}0.007 * * * \\
(0.002)\end{array}$ & $\begin{array}{c}0.008 * * * \\
(0.003)\end{array}$ & $\begin{array}{c}0.006^{* * * *} \\
(0.002)\end{array}$ & $\begin{array}{c}0.007 * * * \\
(0.003)\end{array}$ \\
\hline Tenure sq/100 & $\begin{array}{l}-0.019 \\
(0.016)\end{array}$ & $\begin{array}{l}-0.013 \\
(0.015)\end{array}$ & $\begin{array}{l}-0.014 \\
(0.015)\end{array}$ & $\begin{array}{l}-0.019 \\
(0.015)\end{array}$ & $\begin{array}{l}-0.013 \\
(0.015)\end{array}$ & $\begin{array}{l}-0.014 \\
(0.015)\end{array}$ \\
\hline Overqualified & $\begin{array}{c}0.035 * * * \\
(0.013)\end{array}$ & $\begin{array}{c}0.043 * * * \\
(0.012)\end{array}$ & $\begin{array}{c}0.043 * * * \\
(0.012)\end{array}$ & $\begin{array}{c}0.034 * * * \\
(0.013)\end{array}$ & $\begin{array}{c}0.042 * * * \\
(0.012)\end{array}$ & $\begin{array}{c}0.042 * * * \\
(0.012)\end{array}$ \\
\hline Qualified & $\begin{array}{c}0.053 * * * \\
(0.012)\end{array}$ & $\begin{array}{c}0.053 * * * \\
(0.011)\end{array}$ & $\begin{array}{c}0.054 * * * \\
(0.012)\end{array}$ & $\begin{array}{c}0.052 * * * \\
(0.012)\end{array}$ & $\begin{array}{c}0.052 * * * \\
(0.011)\end{array}$ & $\begin{array}{c}0.052 * * * \\
(0.011)\end{array}$ \\
\hline Female & $\begin{array}{c}-0.024 * * * \\
(0.007)\end{array}$ & $\begin{array}{c}-0.025 * * * \\
(0.007)\end{array}$ & $\begin{array}{c}-0.023 * * * \\
(0.007)\end{array}$ & $\begin{array}{c}-0.023 * * * \\
(0.007)\end{array}$ & $\begin{array}{c}-0.024 * * * \\
(0.007)\end{array}$ & $\begin{array}{c}-0.023 * * * \\
(0.007)\end{array}$ \\
\hline
\end{tabular}

\footnotetext{
${ }^{14}$ Recall that the workforce's skill composition is correlated with the firm's delegation decision (Bloom et al. (2012)).
} 


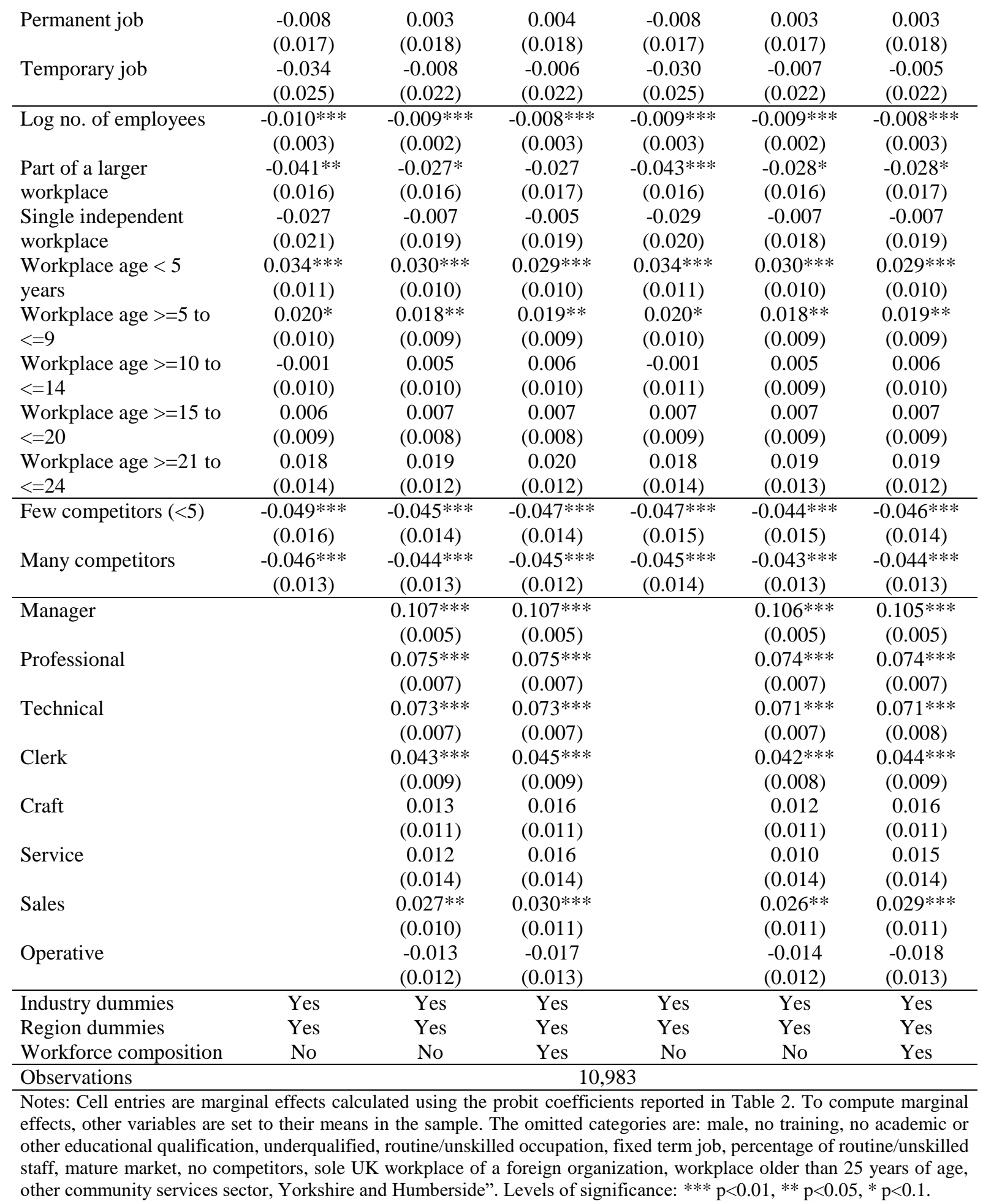

We first discuss the relationship between delegation and training. As seen in column 1, the coefficient for training is positive and statistically significant at the one percent level. When we control for the worker's occupation and the occupational composition at the workplace, the coefficient for training decreases by 31 percent (from 0.228 to 0.155 ) but remains statistically significant at the one percent level. To get a sense of the magnitude of the effect, consider the baseline specification reported in column 1. Accordingly, providing a worker with training is associated, on average, with a 4.1 percent increase in the predicted probability that the worker is granted authority. Once indicators for occupations are added, the positive effect of training 
on the predicted probability of being delegated authority decreases from 4.1 percent to 2.7 percent, as seen in columns 1 and 2 in Table 3.

As indicated, we use a more flexible specification for training in columns 4 through 6 , where six mutually exclusive categories measure the intensity of training provided by the firm. From these results, we observe three patterns. First, as to the effect on being delegated authority, receiving training less than one day is not statistically different from not receiving training at all. Even though the coefficients for obtaining training less than a day are positive, they are not estimated with precision. Second, the probability of delegation monotonically increases with training up to training equal to 5 days, but the coefficients for higher training categories are smaller. However, according to the results in column 4, the coefficients for adjacent training categories for levels of training higher than 5 days are not statistically different from each other. Therefore, a more precise statement to describe the observed relationship is that the probability of delegation monotonically increases with training for levels of training between 1 day and 5 days, and then the positive effect flattens out with additional training. Third, in comparison to the baseline results, the coefficient estimates for training categories become smaller as controls for occupations and the occupational composition at the workplace are added to the estimating equation. ${ }^{15}$

We now turn to the second hypothesis, that is, a firm becomes more likely to delegate authority as the uncertainty in the environment increases. We consider three binary variables indicating whether the market at which the firm currently operates is growing, declining or turbulent (recall that the benchmark category is market being mature). The results provide support for the prediction. In the baseline specifications (columns 1 and 4), operating in a turbulent market, as opposed to operating in a mature market, is positively associated with the probability of delegating authority. The coefficients slightly increase and remain statistically significant (at the five percent level) when controls for occupations are added (see columns 2 and 5). In contrast, the coefficients decrease (by 21 percent) but remain positive and statistically significant at the ten percent level when controls for occupational composition are added (see columns 3 and 6). The results in columns 1 and 3 indicate that operating in a turbulent market, as opposed to operating in a mature market, is associated with 1.9 and 1.5 percent, respectively, higher likelihood of delegation. Turning to other categories, we observe that the coefficients for growing markets are positive in all specifications, but statistically significant (at the ten percent level) only in columns 2 and 5. These coefficients indicate that operating in a growing market, as opposed to in a mature market, is associated with 1.4 percent higher probability of delegation. Finally, even though the coefficients for declining markets are positive in all specifications, they are not statistically different from zero at conventional levels. ${ }^{16}$

The second hypothesis can also be tested by examining the relationship between firm age and delegation. ${ }^{17}$ In their theoretical model, Acemoglu et al. (2007) show that younger firms (and also firms closer to the technological frontier and those operating in more heterogeneous environments) face greater uncertainty regarding optimal business decisions, and therefore, they are more likely to rely on the expertise of their employees rather than using centralized decision-making. Indeed, they also provide supporting evidence for this prediction using data on French and British firms. Recall that our theoretical framework also implies a positive relationship between uncertain environments and the likelihood of delegation. The results in Table 2 indicate that firms under the age of 10 years are more likely to delegate than

\footnotetext{
${ }^{15}$ The only exception to this pattern is training category for 5 to 10 days. This coefficient decreases from 0.292 to 0.149 when controls for occupations are included and then increases to 0.150 when controls for workforce composition are added.

${ }^{16}$ Consistent with our second hypothesis, Avgoustaki (2016) finds that task uncertainty is positively correlated with the worker's discretion over methods he or she uses and the worker's discretion over his or her work schedule.

${ }^{17}$ We thank an anonymous referee for suggesting this discussion.
} 
firms over the age of 25 years. In particular, the likelihood of granting authority to workers in these young firms is around 2-3\% higher (see Table 3). Hence, our finding of a negative relationship between age and delegation also provides support for the second hypothesis.

Turning to the third hypothesis, we first consider the effect of educational attainment on delegation. Columns 1 and 4 show that there is no monotonic relationship between the worker's highest educational attainment and the probability of delegation. More specifically, holding an A-level degree and lower has no effect on delegation (coefficients are small and not statistically different from zero), whereas holding either a college degree or a postgraduate degree, as opposed to holding either a lower educational qualification or no degree at all, is associated with a higher likelihood of delegation. Also, even though the coefficient for holding a postgraduate degree is larger than the coefficient for holding a college degree, the difference is not statistically different from zero. These effects, however, turn insignificant when the worker's occupation is controlled for (see, for example, columns 2 and 3). The reasoning behind this result is the following. Because the variation in educational attainment for a given occupation is not large, the positive effect of holding a college or a postgraduate degree disappears once indicators for occupations enter the specification. Consistent with this reasoning, we also observe that coefficients for certain occupations, such as manager, professional, technical and clerk, are positive and estimated with high precision (all these coefficients are statistically significant at the one percent level).

The results concerning the effect of age and tenure provide support for the third hypothesis. Since in our specifications we include both age and age squared (divided by 100 for convenience) as explanatory variables, the effect of age on the probability of delegation depends on at what age level the marginal effect is evaluated. We observe qualitatively the same result in all specifications: the coefficient for age is positive and the coefficient for its squared term is negative (and both coefficients are statistically significant at the one percent level). This pattern indicates that the probability of delegation increases (at a decreasing rate) with age and the effect turns zero after a certain age. For example, according to the results from column 6, the effect of age on the probability of delegation becomes zero after around age 36 . Similar to age, we include both tenure at firm and its squared term in our estimations. The results show that the coefficient for tenure is positive (and statistically significant at the one percent level) in all specifications, whereas the coefficient for its squared term is negative in all specifications but statistically significant (at the ten percent level) only in columns 2 and $4 .{ }^{18}$ According to the coefficients in columns 2 and 4, the effect of tenure on delegation is positive up to tenure for 20 years. ${ }^{19}$

As indicated, we conjecture that higher match quality between the worker's skills and his or her job is associated with higher probability of delegation. The results provide support for this hypothesis. In all specifications, the coefficients for overqualified and qualified are positive and statistically significant at the one percent level, and the difference between the marginal effects of being overqualified and qualified is statistically significant. ${ }^{20}$ This implies that the effect of being qualified on the probability of delegation is greater than that of being overqualified. According to the results in column 3, being qualified, as opposed to being underqualified, is associated with a 5.4 percent increase in the predicted probability of delegation, whereas being overqualified is associated with a 4.3 percent increase, with respect to the same benchmark, in the predicted probability of delegation.

\footnotetext{
${ }^{18}$ We also estimated our richest specification (column 6) by excluding tenure squared. In that case, the coefficient for tenure turns out to be 0.019 and statistically significant at the one percent level.

${ }^{19}$ Consistent with this result, Lo et al. (2016) examine data on sales people and find that longer tenure at the job is associated with higher likelihood of delegating pricing authority to employees.

${ }^{20}$ The p-values associated with these tests are lower than 0.01 in all specifications.
} 
As our delegation measure is derived from a question in which potential responses have a meaningful (ordinal) ranking, we also estimate an ordered probit model where the dependent variable describes the degree of delegation the worker has been granted with four distinct categories. The results are reported in Table 4. Note that specifications in columns 1 through 4 of Table 4 are analogous to those in columns 3 through 6 of Table 2, respectively.

Table 4 Determinants of Delegation

\begin{tabular}{|c|c|c|c|c|}
\hline VARIABLES & $(1)$ & $(2)$ & (3) & (4) \\
\hline Training & $\begin{array}{c}0.086 * * * \\
(0.024)\end{array}$ & & & \\
\hline \multicolumn{5}{|l|}{ Training } \\
\hline$<1$ day & & $\begin{array}{c}-0.056 \\
(0.037)\end{array}$ & $\begin{array}{c}-0.047 \\
(0.037)\end{array}$ & $\begin{array}{c}-0.044 \\
(0.037)\end{array}$ \\
\hline$>=1$ day to $<2$ days & & $\begin{array}{c}0.136 * * * \\
(0.034)\end{array}$ & $\begin{array}{c}0.083 * * \\
(0.034)\end{array}$ & $\begin{array}{c}0.083 * * \\
(0.034)\end{array}$ \\
\hline$>=2$ days to $<5$ days & & $\begin{array}{c}0.237 * * * \\
(0.032)\end{array}$ & $\begin{array}{c}0.151 * * * \\
(0.033)\end{array}$ & $\begin{array}{c}0.151 * * * \\
(0.033)\end{array}$ \\
\hline$>=5$ days to $<10$ days & & $\begin{array}{c}0.238 * * * \\
(0.040)\end{array}$ & $\begin{array}{c}0.113 * * * \\
(0.041)\end{array}$ & $\begin{array}{c}0.116^{* * *} * \\
(0.041)\end{array}$ \\
\hline$>=10$ days & & $\begin{array}{c}0.206^{* * *} * \\
(0.045)\end{array}$ & $\begin{array}{c}0.118 * * * \\
(0.045)\end{array}$ & $\begin{array}{c}0.120 * * * \\
(0.044)\end{array}$ \\
\hline Growing market & $\begin{array}{l}0.059^{*} \\
(0.034)\end{array}$ & $\begin{array}{l}0.063^{*} \\
(0.034)\end{array}$ & $\begin{array}{c}0.069 * * \\
(0.034)\end{array}$ & $\begin{array}{l}0.059^{*} \\
(0.034)\end{array}$ \\
\hline Declining market & $\begin{array}{c}0.036 \\
(0.056)\end{array}$ & $\begin{array}{c}0.045 \\
(0.057)\end{array}$ & $\begin{array}{c}0.054 \\
(0.055)\end{array}$ & $\begin{array}{c}0.036 \\
(0.056)\end{array}$ \\
\hline Turbulent market & $\begin{array}{c}0.037 \\
(0.038) \\
\end{array}$ & $\begin{array}{c}0.042 \\
(0.038) \\
\end{array}$ & $\begin{array}{c}0.047 \\
(0.037) \\
\end{array}$ & $\begin{array}{c}0.039 \\
(0.038) \\
\end{array}$ \\
\hline GCSE grades D-G & $\begin{array}{c}0.013 \\
(0.043)\end{array}$ & $\begin{array}{c}-0.046 \\
(0.042)\end{array}$ & $\begin{array}{c}0.016 \\
(0.043)\end{array}$ & $\begin{array}{c}0.012 \\
(0.043)\end{array}$ \\
\hline GCSE grades A-C & $\begin{array}{c}-0.121 * * * \\
(0.029)\end{array}$ & $\begin{array}{c}-0.116^{* * *} \\
(0.029)\end{array}$ & $\begin{array}{c}-0.118 * * * \\
(0.029)\end{array}$ & $\begin{array}{c}-0.119 * * * \\
(0.029)\end{array}$ \\
\hline A-levels & $\begin{array}{c}-0.020 \\
(0.039)\end{array}$ & $\begin{array}{c}0.027 \\
(0.039)\end{array}$ & $\begin{array}{l}-0.022 \\
(0.039)\end{array}$ & $\begin{array}{c}-0.020 \\
(0.039)\end{array}$ \\
\hline First degree & $\begin{array}{c}-0.027 \\
(0.034)\end{array}$ & $\begin{array}{c}0.090 * * * \\
(0.033)\end{array}$ & $\begin{array}{c}-0.033 \\
(0.034)\end{array}$ & $\begin{array}{l}-0.026 \\
(0.034)\end{array}$ \\
\hline Higher degree & $\begin{array}{l}0.096 * \\
(0.054)\end{array}$ & $\begin{array}{c}0.200^{* * *} * \\
(0.054)\end{array}$ & $\begin{array}{c}0.084 \\
(0.054)\end{array}$ & $\begin{array}{l}0.099 * \\
(0.054)\end{array}$ \\
\hline Age & $\begin{array}{c}0.034 * * * \\
(0.006)\end{array}$ & $\begin{array}{c}0.047 * * * \\
(0.006)\end{array}$ & $\begin{array}{c}0.033 * * * \\
(0.006)\end{array}$ & $\begin{array}{c}0.034 * * * \\
(0.006)\end{array}$ \\
\hline Age sq/100 & $\begin{array}{c}-0.037 * * * \\
(0.008)\end{array}$ & $\begin{array}{c}-0.053^{* * *} * \\
(0.008)\end{array}$ & $\begin{array}{c}-0.036 * * * \\
(0.008)\end{array}$ & $\begin{array}{c}-0.037 * * * \\
(0.008)\end{array}$ \\
\hline Tenure & $\begin{array}{c}0.043 * * * \\
(0.008)\end{array}$ & $\begin{array}{c}0.046 * * * \\
(0.008)\end{array}$ & $\begin{array}{c}0.042 * * * \\
(0.008)\end{array}$ & $\begin{array}{c}0.043 * * * \\
(0.008)\end{array}$ \\
\hline Tenure sq/100 & $\begin{array}{c}-0.124 * * * \\
(0.037)\end{array}$ & $\begin{array}{c}-0.133 * * * \\
(0.036)\end{array}$ & $\begin{array}{c}-0.118 * * * \\
(0.036)\end{array}$ & $\begin{array}{c}-0.125 * * * \\
(0.036)\end{array}$ \\
\hline Overqualified & $\begin{array}{c}0.286 * * * \\
(0.045)\end{array}$ & $\begin{array}{c}0.238 * * * \\
(0.046)\end{array}$ & $\begin{array}{c}0.284 * * * \\
(0.045)\end{array}$ & $\begin{array}{c}0.287 * * * \\
(0.045)\end{array}$ \\
\hline Qualified & $\begin{array}{c}0.329 * * * \\
(0.047)\end{array}$ & $\begin{array}{c}0.287 * * * \\
(0.047)\end{array}$ & $\begin{array}{c}0.319 * * * \\
(0.047)\end{array}$ & $\begin{array}{c}0.325^{* * * *} \\
(0.047)\end{array}$ \\
\hline Female & $\begin{array}{c}-0.107 * * * \\
(0.027)\end{array}$ & $\begin{array}{c}-0.114 * * * * \\
(0.027)\end{array}$ & $\begin{array}{c}-0.104 * * * \\
(0.027)\end{array}$ & $\begin{array}{c}-0.102 * * * \\
(0.027)\end{array}$ \\
\hline Permanent job & $\begin{array}{c}0.027 \\
(0.069)\end{array}$ & $\begin{array}{l}-0.006 \\
(0.066)\end{array}$ & $\begin{array}{c}0.029 \\
(0.068)\end{array}$ & $\begin{array}{c}0.024 \\
(0.069)\end{array}$ \\
\hline Temporary job & $\begin{array}{c}-0.095 \\
(0.083)\end{array}$ & $\begin{array}{c}-0.156^{*} \\
(0.080)\end{array}$ & $\begin{array}{l}-0.079 \\
(0.083)\end{array}$ & $\begin{array}{l}-0.087 \\
(0.083)\end{array}$ \\
\hline Log no. of employees & $\begin{array}{c}-0.040 * * * \\
(0.011)\end{array}$ & $\begin{array}{c}-0.045^{* * *} \\
(0.010)\end{array}$ & $\begin{array}{c}-0.045^{* * *} * \\
(0.010)\end{array}$ & $\begin{array}{c}-0.041 * * * \\
(0.011)\end{array}$ \\
\hline
\end{tabular}




\begin{tabular}{|c|c|c|c|c|}
\hline Part of a larger workplace & $\begin{array}{c}0.072 \\
(0.057)\end{array}$ & $\begin{array}{c}0.017 \\
(0.055)\end{array}$ & $\begin{array}{c}0.075 \\
(0.056)\end{array}$ & $\begin{array}{c}0.062 \\
(0.055)\end{array}$ \\
\hline Single independent workplace & $\begin{array}{c}0.178 * * * \\
(0.062)\end{array}$ & $\begin{array}{c}0.096 \\
(0.059)\end{array}$ & $\begin{array}{c}0.182 * * * \\
(0.061)\end{array}$ & $\begin{array}{c}0.168 * * * \\
(0.061)\end{array}$ \\
\hline Workplace age $<5$ years & $\begin{array}{c}0.171 * * * \\
(0.057)\end{array}$ & $\begin{array}{c}0.183 * * * \\
(0.062)\end{array}$ & $\begin{array}{c}0.167 * * * \\
(0.059)\end{array}$ & $\begin{array}{c}0.171 * * * \\
(0.057)\end{array}$ \\
\hline Workplace age $>=5$ to $<=9$ & $\begin{array}{c}0.123 * * * \\
(0.039)\end{array}$ & $\begin{array}{c}0.141 * * * \\
(0.041)\end{array}$ & $\begin{array}{c}0.124 * * * \\
(0.040)\end{array}$ & $\begin{array}{c}0.123 * * * \\
(0.040)\end{array}$ \\
\hline Workplace age $>=10$ to $<=14$ & $\begin{array}{c}0.091 * * \\
(0.045)\end{array}$ & $\begin{array}{l}0.077 * \\
(0.046)\end{array}$ & $\begin{array}{c}0.098 * * \\
(0.046)\end{array}$ & $\begin{array}{c}0.092 * * \\
(0.046)\end{array}$ \\
\hline Workplace age $>=15$ to $<=20$ & $\begin{array}{c}0.063 \\
(0.039)\end{array}$ & $\begin{array}{l}0.068^{*} \\
(0.040)\end{array}$ & $\begin{array}{l}0.069 * \\
(0.038)\end{array}$ & $\begin{array}{l}0.066^{*} \\
(0.039)\end{array}$ \\
\hline Workplace age $>=21$ to $<=24$ & $\begin{array}{c}0.189 * * * \\
(0.058)\end{array}$ & $\begin{array}{c}0.183 * * * \\
(0.058)\end{array}$ & $\begin{array}{c}0.192 * * * \\
(0.059)\end{array}$ & $\begin{array}{c}0.189 * * * \\
(0.059)\end{array}$ \\
\hline Few competitors $(<5)$ & $\begin{array}{l}-0.066 \\
(0.053)\end{array}$ & $\begin{array}{l}-0.049 \\
(0.054)\end{array}$ & $\begin{array}{l}-0.039 \\
(0.055)\end{array}$ & $\begin{array}{l}-0.063 \\
(0.053)\end{array}$ \\
\hline Many competitors & $\begin{array}{l}-0.035 \\
(0.052)\end{array}$ & $\begin{array}{l}-0.008 \\
(0.053)\end{array}$ & $\begin{array}{l}-0.010 \\
(0.054)\end{array}$ & $\begin{array}{l}-0.032 \\
(0.052)\end{array}$ \\
\hline Manager & $\begin{array}{c}0.886 * * * \\
(0.054)\end{array}$ & & $\begin{array}{c}0.836^{* * * *} \\
(0.053)\end{array}$ & $\begin{array}{c}0.859 * * * \\
(0.055)\end{array}$ \\
\hline Professional & $\begin{array}{c}0.345^{* * * *} \\
(0.053)\end{array}$ & & $\begin{array}{c}0.288 * * * \\
(0.051)\end{array}$ & $\begin{array}{c}0.328 * * * \\
(0.053)\end{array}$ \\
\hline Technical & $\begin{array}{c}0.478 * * * \\
(0.051)\end{array}$ & & $\begin{array}{c}0.427 * * * \\
(0.049)\end{array}$ & $\begin{array}{c}0.459 * * * \\
(0.051)\end{array}$ \\
\hline Clerk & $\begin{array}{c}0.182 * * * \\
(0.050)\end{array}$ & & $\begin{array}{c}0.134 * * * \\
(0.049)\end{array}$ & $\begin{array}{c}0.170 * * * \\
(0.050)\end{array}$ \\
\hline Craft & $\begin{array}{c}0.031 \\
(0.057)\end{array}$ & & $\begin{array}{c}0.001 \\
(0.054)\end{array}$ & $\begin{array}{c}0.021 \\
(0.057)\end{array}$ \\
\hline Service & $\begin{array}{c}0.104 \\
(0.069)\end{array}$ & & $\begin{array}{c}0.087 \\
(0.064)\end{array}$ & $\begin{array}{c}0.089 \\
(0.069)\end{array}$ \\
\hline Sales & $\begin{array}{c}0.000 \\
(0.058)\end{array}$ & & $\begin{array}{l}-0.025 \\
(0.054)\end{array}$ & $\begin{array}{c}-0.003 \\
(0.058)\end{array}$ \\
\hline Operative & $\begin{array}{c}-0.106 * * \\
(0.053)\end{array}$ & & $\begin{array}{c}-0.088^{*} \\
(0.049)\end{array}$ & $\begin{array}{c}-0.114 * * \\
(0.053)\end{array}$ \\
\hline Industry dummies & Yes & Yes & Yes & Yes \\
\hline Region dummies & Yes & Yes & Yes & Yes \\
\hline Workforce composition & Yes & No & No & Yes \\
\hline Ordered probit cutoffs & & & & \\
\hline Cutoff1 & $\begin{array}{c}0.065 \\
(0.190)\end{array}$ & $\begin{array}{c}0.072 \\
(0.175)\end{array}$ & $\begin{array}{c}0.070 \\
(0.179)\end{array}$ & $\begin{array}{c}0.042 \\
(0.189)\end{array}$ \\
\hline Cutoff2 & $\begin{array}{c}0.659 * * * \\
(0.190)\end{array}$ & $\begin{array}{c}0.649 * * * \\
(0.175)\end{array}$ & $\begin{array}{c}0.663 * * * \\
(0.179)\end{array}$ & $\begin{array}{c}0.636^{* * * *} \\
(0.189)\end{array}$ \\
\hline Cutoff3 & $\begin{array}{c}1.707 * * * \\
(0.191)\end{array}$ & $\begin{array}{c}1.669 * * * \\
(0.176)\end{array}$ & $\begin{array}{c}1.711 * * * \\
(0.179)\end{array}$ & $\begin{array}{c}1.685 * * * \\
(0.190)\end{array}$ \\
\hline
\end{tabular}

Notes: Cell entries are ordered probit coefficients with standard errors clustered at the workplace level. The omitted categories are: male, no training, no academic or other educational qualification, underqualified, routine/unskilled occupation, fixed term job, percentage of routine/unskilled staff, mature market, no competitors, sole UK workplace of a foreign organization, workplace older than 25 years of age, other community services sector, Yorkshire and Humberside". Levels of significance: *** $\mathrm{p}<0.01, * * \mathrm{p}<0.05, *$ $\mathrm{p}<0.1$.

The results concerning the relationship between training and delegation remain consistent with the first hypothesis. When training is measured by a binary variable, the coefficient is positive and statistically significant at the one percent level (see column 1). This means that the intensity of delegation (which is modelled as a latent variable) increases with training. The incremental effect of obtaining training (i.e., when training increases from 0 to 1 ) is associated with a 9.2 percentage points decrease in the probability that delegation is not 
granted (i.e., the worker's response is "None"). ${ }^{21}$ In columns 2 through 4 , we observe that the relationship between obtaining training less than one day and delegation is not statistically significant, whereas obtaining training more than one day is positively related with training. Further, the coefficient for training equal to 1 to 2 days is smaller than the coefficient for training equal to 2 to 5 days in all specifications, but the difference is statistically significant only in columns 2 and $3 .^{22}$ Finally, adjacent training categories for levels of training higher than 5 days are not statistically different from each other.

Next, we look at the effect of the uncertainty in the environment, captured by indicators for the state of the market. Consistent with the earlier results, the coefficients for these indicator variables remain positive, as predicted by the theory, but not all of them are statistically significant. We observe the following differences between the results from Table 4 and those from Table 2. First, the coefficients for operating in a turbulent market remain positive, but become statistically not different from zero (this happens because the size of the coefficient decreases by approximately 50 percent, whereas the standard error decreases only marginally). Second, despite being marginally smaller than in the earlier set of results, the effect of operating in a growing market is positive and statistically significant (at the ten percent level in columns 1,2 , and 4 and at the five percent level in column 3 ).

Finally, we turn to how delegation is related to our proxies for the precision of the worker's signal. We observe that the qualitative results concerning age and tenure at the firm are the same. That is, the main effect is positive, whereas the coefficient for the quadratic term is negative (both coefficients are significant at the one percent level in all specifications). Concerning the effect of educational attainment, we observe the same pattern except for holding a GCSE degree (grades AC). More precisely, we observe that holding a college degree and holding a postgraduate degree are associated with a higher likelihood of delegation (see column 1); these effects, however, disappear once we control for occupational dummies (as we observe in the probit model). The difference from the earlier set of results is that the coefficient for GCSE degree (grades A-C) turn significant (at the one percent level) in ordered probit models. Last, we obtain qualitatively the same results concerning the effect of the match quality between the worker's skills and his or her job. Overall, the results suggest that the correlation between being either qualified or overqualified and delegation is positive. ${ }^{23}$

\section{Conclusion}

This paper uses a cross section of matched employer-employee data from Britain to examine the determinants of delegation. Using the existing theories, particularly the analysis in Ekinci and Theodoropoulos (2021), we formulate three hypotheses that we take to data. Our first hypothesis focuses on the firm's incentives to facilitate information acquisition by the worker and it predicts that firm-provided training and delegation are positively related. Next, we consider the factors that determine the informational value of delegating authority to employees. As predicted by the existing theories in the literature, our second and third hypotheses assert, respectively, that firms become more likely to delegate authority as the uncertainty in the environment increases and as the worker's information becomes more precise.

\footnotetext{
${ }^{21}$ The incremental effect is statistically significant at the one percent level $(z=3.33)$.

${ }^{22}$ More specifically, the p-value for the difference between training categories "one to two days" and "two to five days" is 0.060 in column 2 and 0.630 in column 3.

${ }^{23}$ Note that the coefficient for qualified is greater than the coefficient for overqualified, but the difference is significant at modest levels. Specifically, the p-values from the corresponding tests are $0.122,0.092$ and 0.061 in columns 2,3 , and 4, respectively.
} 
The empirical results provide strong support for the first hypothesis. More specifically, we find that both training incidence and intensity are associated with higher likelihood of delegation. Consistent with the second hypothesis, the results suggest that higher degrees of uncertainty, captured by the current state of the product market, induce firms to delegate more frequently. Finally, we find that workers with higher educational attainment and age, longer tenure at the firm, and workers whose skills match better their jobs' requirements are also more likely to be granted authority by their employers. 


\section{References}

Acemoglu, D., Aghion, P., Lelarge, C., Van Reenen, J., and Zilibotti, F. (2007). “Technology, information, and the decentralization of the firm," The Quarterly Journal of Economics, 122(4), 1759-1799.

Aghion, P. and Tirole, J. (1997). "Formal and real authority in organizations," Journal of Political Economy, 105(1), 1-29.

Aghion, P., Bloom, N., and Van Reenen, J. (2013). "Incomplete contracts and the internal organization of firms," The Journal of Law, Economics, \& Organization, 30 (suppl_1), i37-i63.

Avgoustaki, A. (2016). "Work uncertainty and extensive work effort: The mediating role of human resource practices," ILR Review, 69(3), 656-682.

Baker, G., Gibbons, R., and Murphy, K. J. (1999). "Informal authority in organizations," The Journal of Law, Economics, \& Organization, 15(1), 56-73.

Bilanakos, C., Heywood, J. S., Sessions, J. G., and Theodoropoulos, N. (2018). "Does delegation increase worker training?," Economic Inquiry, 56(2), 1089-1115.

Bloom, N., Sadun, R., and Van Reenen, J. (2012). "The organization of firms across countries," The Quarterly Journal of Economics, 127(4), 1663-1705.

Bolton, P. and Dewatripont, M., (2013). "Authority in Organizations," in R. Gibbons and J. Roberts (ed.), Handbook of Organizational Economics: 342-372. New Jersey: Princeton University Press.

Chaplin, J., Mangla, J., Purdon, S., and Airey, C. (2005). The workplace employment relations survey (WERS) 2004 technical report. London: National Centre for Official Research.

Che, Y. K. and Kartik, N. (2009). "Opinions as incentives," Journal of Political Economy, 117(5), 815-860.

DeVaro, J. and Kurtuluş, F. A. (2010). "An empirical analysis of risk, incentives and the delegation of worker authority," ILR Review, 63(4), 641-661.

De Varo, J. and Prasad, S. (2015). "The relationship between delegation and incentives across occupations: Evidence and theory," The Journal of Industrial Economics, 63(2), 279312.

Ekinci, E. and Theodoropoulos, N. (2021). "Disagreement and informal delegation in organizations," International Journal of Industrial Organization, 74, 102696.

Foss, N. J. and Laursen, K. (2005). "Performance pay, delegation and multitasking under uncertainty and innovativeness: An empirical investigation," Journal of Economic Behavior \& Organization, 58(2), 246-276.

Gibbons, R., Matouschek, N., and Roberts, J., (2013). "Decisions in Organizations," in R. Gibbons and J. Roberts (ed.), Handbook of Organizational Economics: 373-431. New Jersey: Princeton University Press.

Lo, D., Dessein, W., Ghosh, M., and Lafontaine, F. (2016). "Price delegation and performance pay: Evidence from industrial sales forces," The Journal of Law, Economics, and Organization, 32(3), 508-544.

Prendergast, C. (2002). "The tenuous trade-off between risk and incentives," Journal of Political Economy, 110(5), 1071-1102. 